\title{
Dark matter detection, Standard Model parameters and Intermediate Scale Supersymmetry
}

\author{
David Dunsky, ${ }^{a, b}$ Lawrence J. Hall ${ }^{a, b}$ and Keisuke Harigaya ${ }^{c}$ \\ ${ }^{a}$ Department of Physics, University of California, \\ Berkeley, CA 94720, U.S.A. \\ ${ }^{b}$ Theoretical Physics Group, Lawrence Berkeley National Laboratory, \\ Berkeley, CA 94720, U.S.A. \\ ${ }^{c}$ School of Natural Sciences, Institute for Advanced Study, \\ Princeton, NJ 08540, U.S.A. \\ E-mail: ddunsky@berkeley.edu, ljh@berkeley.edu, \\ keisukeharigaya@ias.edu
}

ABSTRACT: The vanishing of the Higgs quartic coupling at a high energy scale may be explained by Intermediate Scale Supersymmetry, where supersymmetry breaks at $\left(10^{9}\right.$ $\left.10^{12}\right) \mathrm{GeV}$. The possible range of supersymmetry breaking scales can be narrowed down by precise measurements of the top quark mass and the strong coupling constant. On the other hand, nuclear recoil experiments can probe Higgsino or sneutrino dark matter up to a mass of $10^{12} \mathrm{GeV}$. We derive the correlation between the dark matter mass and precision measurements of standard model parameters, including supersymmetric threshold corrections. The dark matter mass is bounded from above as a function of the top quark mass and the strong coupling constant. The top quark mass and the strong coupling constant are bounded from above and below respectively for a given dark matter mass. We also discuss how the observed dark matter abundance can be explained by freeze-out or freeze-in during a matter-dominated era after inflation, with the inflaton condensate being dissipated by thermal effects.

Keywords: Cosmology of Theories beyond the SM, Higgs Physics, Quark Masses and SM Parameters, Supersymmetric Standard Model

ARXIV EPRINT: 2011.12302 


\section{Contents}

1 Introduction 1

2 The tree-level boundary condition on the SM quartic coupling 4

3 Direct detection of dark matter 5

3.1 Higgsino or sneutrino dark matter 5

3.1.1 Higgsino dark matter 5

$\begin{array}{lll}\text { 3.1.2 Sneutrino dark matter } & 6\end{array}$

$\begin{array}{ll}3.2 & \text { Direct detection rate and standard model parameters }\end{array}$

4 Including threshold corrections to the Higgs quartic $\quad 7$

5 Supersymmetry breaking constrained by unification 10

6 Cosmological abundance of LSP with intermediate scale mass $\quad 13$

$\begin{array}{lll}\text { 6.1 Direct decay of the inflaton } & 14\end{array}$

6.2 Production during the inflaton dominated era 14

$\begin{array}{lll}6.3 & \text { Other possibilities } & 16\end{array}$

$\begin{array}{lll}7 & \text { Conclusions } & 17\end{array}$

$\begin{array}{lr}\text { A Stability bound on a trilinear coupling } & 18\end{array}$

\section{Introduction}

In 1985, Goodman and Witten proposed that halo dark matter could be detected directly in terrestrial experiments by observing small energy depositions from elastic scattering of dark matter particles from nuclei [1]. Their first illustration was of a neutral particle, such as a heavy neutrino, scattering via $t$-channel $Z$ exchange with a cross section per nucleon of order $\sigma v \sim G_{F}^{2} \mu_{\text {red }}^{2} / 2 \pi$, where $\mu_{\text {red }}$ is the reduced mass of the dark matter and nucleon. They computed a signal of order $10^{2}-10^{4}$ events per Kg per day for dark matter masses in the $\mathrm{GeV}$ to $\mathrm{TeV}$ range, depending on nuclear target. In the intervening 35 years, a succession of ever larger and more sensitive detectors have excluded this example by many orders of magnitude, so that the focus has shifted to theories where there is no contribution to the scattering from tree-level weak interactions. In fact, as the number density of dark matter particles scales as the inverse of its mass, present data constrains the mass of dark matter with tree-level $Z$ exchange to be larger than $3 \times 10^{9} \mathrm{GeV}[2]$. Proposed detectors [3-5] will probe the mass range

$$
M_{\mathrm{DM}, Z \text {-exchange }}=\left(3 \times 10^{9}-2 \times 10^{12}\right) \mathrm{GeV} .
$$


The discovery of the Higgs boson at the Large Hadron Collider (LHC) completes the Standard Model (SM). Electroweak symmetry breaking arises from the potential

$$
V_{\mathrm{SM}}(H)=-m^{2}|H|^{2}+\lambda|H|^{4},
$$

via the ground state value of the Higgs field $\langle H\rangle=v \simeq 174 \mathrm{GeV}$. The Higgs boson mass is $m_{h}^{2}=4 \lambda v^{2}$. No other new particles have been discovered at the LHC so far, and in this paper we assume that the SM is valid to very high energies. All the SM couplings can be computed at high energies to high precision, including the Higgs quartic coupling [6]. As shown in figure 1, this running indicates that the Higgs quartic coupling vanishes at the scale

$$
\mu_{\lambda}=10^{9-12} \mathrm{GeV}
$$

which we call the Higgs quartic scale. Indeed, within the context of the SM as an effective field theory to very high energies, a key result of the LHC is the discovery of this new mass scale. In this paper we assume that physics beyond the SM first appears at $\mu_{\lambda}$, and the form of the new physics explains why the Higgs quartic is so small at this scale. It is interesting to note that, if dark matter couples to the weak interaction, the recent direct detection experiments have started to explore dark matter masses in the vicinity of the Higgs quartic scale. The mass range to be explored by the next generation of experiments, (1.1), will probe the entire range of (1.3).

Since the discovery of a Higgs with mass of $125 \mathrm{GeV}$, several proposals have been made for physics at $\mu_{\lambda}$ that explains the small quartic coupling, including supersymmetry [7-9], extra dimension [10], Peccei-Quinn symmetry [11], and Higgs Parity symmetry [12-16]. In this paper we pursue the case of Intermediate Scale Supersymmetry (ISS), where the superpartner mass scale $\tilde{m}$ is of order the Higgs quartic scale. The identification of $\mu_{\lambda}$ with $\tilde{m}$ is natural $[7,8]$ since supersymmetry predicts a very small SM Higgs quartic at the scale $\tilde{m}$ for a wide range of supersymmetry breaking parameters. Unlike in $[7,8]$, we study the case of Higgsino or sneutrino Lightest Supersymmetric Particle (LSP) dark matter with mass of order $\tilde{m}$, since this gives a direct detection signal that is correlated with the Higgs quartic scale.

In this paper, we examine the correlation in ISS between the dark matter detection signal via $Z$ exchange and the precision measurement of the top quark mass, $m_{t}$, the strong coupling constant, $\alpha_{s}\left(m_{Z}\right)$, (and to a lesser extent, of the Higgs boson mass, $m_{h}$ ). A dark matter signal will determine the mass of the LSP and precision measurements will greatly reduce the uncertainties in the Higgs quartic scale. In particular, we find that the discovery of a direct detection signal implies an upper bound on the top quark mass and a lower bound on the strong coupling constant. The effects on the running of the Higgs quartic in reducing the uncertainties in $m_{t}, \alpha_{s}\left(m_{Z}\right)$ and $m_{h}$ are shown by the colored bands in figure 1. Future uncertainties in $m_{t}(0.01 \mathrm{GeV}), \alpha_{s}\left(m_{Z}\right)(0.0001)$, and $m_{h}(0.01 \mathrm{GeV})$ from measurements at future lepton colliders [17-21], improved lattice calculations [22], and the high-luminosity LHC [23], will substantially reduce the uncertainty in $\mu_{\lambda}$ to within a few tens of percents, as shown by the solid black strip in figure 1 which is centered at the current central values of $m_{\mathrm{t}}, \alpha_{s}\left(m_{Z}\right)$, and $m_{h}$. 

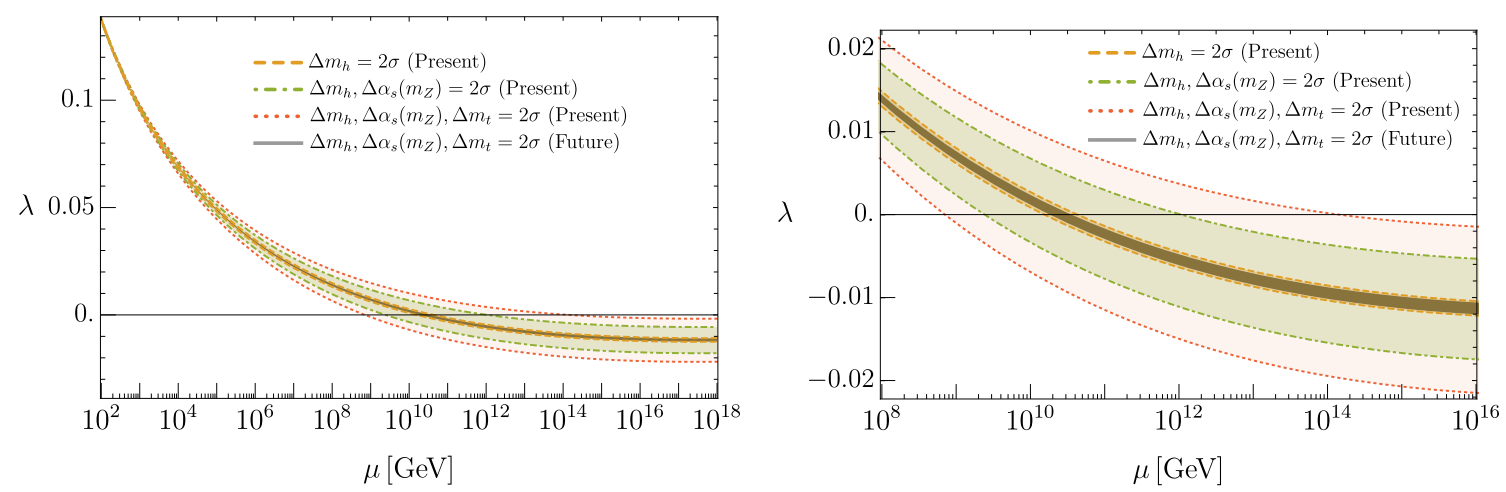

Figure 1. Running of the SM quartic coupling with current and future uncertainties in $m_{t}, \alpha_{s}\left(m_{Z}\right)$, and $m_{h}$. Their central values are $m_{t}=172.76 \mathrm{GeV}, \alpha_{s}\left(m_{Z}\right)=0.1179$, and $m_{h}=125.10 \mathrm{GeV}$.

In 1977, Lee and Weinberg showed dark matter, if coupled to the weak interaction, could be produced in the early universe by freezing-out, losing thermal equilibrium while non-relativistic [24]. Indeed, they discovered that a heavy neutrino, with a $\mathrm{GeV}$-scale mass, could yield the observed dark matter abundance. Many other electroweak dark matter candidates arising from freeze-out were studied, with masses up to several TeV. Apparently our proposal of Higgsino or sneutrino dark matter with a mass of $10^{9}-10^{12} \mathrm{GeV}$ leads to a huge overproduction of dark matter. However, we find that the observed abundance can result from freeze-out or freeze-in during a matter-dominated era after inflation. The inflaton mass must be below the dark matter mass, otherwise the $O(1)$ branching fraction of the inflaton into sparticles leads to an overproduction of dark matter. Then during freeze-out or freeze-in, the inflaton is dissipated by scattering reactions rather than by decays. If the products of the scattering reactions are thermalized at a high enough temperature, freeze-out occurs; otherwise, the abundance is set by freeze-in from non-thermal radiation. Either way, determining the dark matter mass from direct detection will provide a correlation between the reheat temperature after inflation and the inflaton mass.

In section 2, building on $[7,8]$, we show that if the UV completion of the SM EFT is provided by ISS, there is a large region of parameter space where the SM quartic coupling is predicted to be very small at $\tilde{m}$, and hence $\tilde{m} \sim \mu_{\lambda}$. In section 3 we compute the present limits on Higgsino and sneutrino dark matter, and compute the reaches expected for XENONnT, LZ, and DARWIN. We then study the correlation between the dark matter signal and future precision measurements of $m_{t}, \alpha_{s}\left(m_{Z}\right)$, and $m_{h}$. In section 4 we study how this correlation is affected by supersymmetric threshold corrections to the Higgs quartic coupling in the Minimal Supersymmetric Standard Model (MSSM). We find that these threshold corrections can be significant and derive an upper bound on the Higgsino or sneutrino LSP mass as a function of the top quark mass and the strong coupling constant. An observable direct detection signal is predicted for top masses above a critical value. In section 5 we compute the supersymmetric threshold corrections in a scheme where the supersymmetry breaking parameters are constrained to a universal form at unified scales. In section 6 we compute the relic dark matter abundance from freeze-out or freeze-in during 
a matter dominated era where the inflaton condensate is dissipated by scattering reactions. Finally, we draw conclusions in section 7.

\section{The tree-level boundary condition on the SM quartic coupling}

We take the SM to be the effective theory below the scale of supersymmetry breaking, $\tilde{m}$. In this section, we review the tree-level prediction for the SM Higgs quartic coupling, $\lambda_{\text {tree }}$. At scale $\tilde{m}$, we assume that there is no gauge symmetry breaking and the theory contains a single pair of Higgs doublets, $\left(H_{u}, H_{d}\right)$, and no weak singlets or triplets which have a zero hypercharge and couple to the Higgs doublets. For a wide range of parameters of this Higgs sector, we find $\lambda(\tilde{m}) \ll 0.01$; remarkably there are large regions with $\lambda(\tilde{m}) \lesssim 0.001$, and the supersymmetry breaking scale $\tilde{m}$ may be identified with the Higgs quartic scale $\mu_{\lambda}$.

The Higgs potential is

$$
\begin{aligned}
V\left(H_{u}, H_{d}\right)= & \left(\mu^{2}+m_{H_{u}}^{2}\right) H_{u}^{\dagger} H_{u}+\left(\mu^{2}+m_{H_{d}}^{2}\right) H_{d}^{\dagger} H_{d}+\left(B \mu H_{u} H_{d}+\text { h.c. }\right) \\
& +\frac{g^{2}}{8}\left(H_{u}^{\dagger} \vec{\sigma} H_{u}+H_{d}^{\dagger} \vec{\sigma} H_{d}\right)^{2}+\frac{g^{\prime 2}}{8}\left(H_{u}^{\dagger} H_{u}-H_{d}^{\dagger} H_{d}\right)^{2},
\end{aligned}
$$

where $\mu$ is the supersymmetric Higgs mass parameter, while $m_{H_{u}}^{2}, m_{H_{d}}^{2}$, and $B \mu$ are supersymmetry-violating mass parameters. These parameters are all taken real, without loss of generality, and have sizes determined by the scale of supersymmetry breaking, $\tilde{m}$. The constants $g$ and $g^{\prime}$ are the $\mathrm{SU}(2)$ and $\mathrm{U}(1)$ gauge couplings. Requiring electroweak symmetry to be unbroken at $\tilde{m}$ and one combination of the Higgs doublets to be much lighter than $\tilde{m}$ requires that $\mu^{2}+m_{H_{u, d}}^{2}$ are both positive. The fine tune for a light doublet requires that $B \mu$ is taken to be the geometric mean of $\mu^{2}+m_{H_{u, d}}^{2}$. The light SM Higgs doublet is

$$
H=\sin \beta H_{u}+\cos \beta H_{d}^{\dagger},
$$

where $\tan ^{2} \beta=\left(\mu^{2}+m_{H_{d}}^{2}\right) /\left(\mu^{2}+m_{H_{u}}^{2}\right)$, and we take $\beta$ in the first quadrant.

Matching the two theories at $\tilde{m}$ gives the tree-level value for $\lambda(\tilde{m})$

$$
\lambda(\tilde{m})_{\text {tree }}=\frac{g_{2}(\tilde{m})^{2}+g^{\prime}(\tilde{m})^{2}}{8} \cos ^{2} 2 \beta
$$

with

$$
\cos ^{2} 2 \beta=\left(\frac{m_{H_{u}}^{2}-m_{H_{d}}^{2}}{m_{H_{u}}^{2}+m_{H_{d}}^{2}+2 \mu^{2}}\right)^{2} .
$$

ISS gives $0 \leq \lambda(\tilde{m})_{\text {tree }} \leq\left(g_{2}(\tilde{m})^{2}+g^{\prime}(\tilde{m})^{2}\right) / 8 \simeq 0.06$ and hence at tree level $\tilde{m} \lesssim \mu_{\lambda}$. Furthermore, over a wide range of values for $m_{H_{u}}^{2}, m_{H_{d}}^{2}$, and $\mu$ the $\cos ^{2} 2 \beta$ factor gives a significant further suppression of $\lambda(\tilde{m})_{\text {tree }}$, as shown in figure 2 . Indeed, $\cos 2 \beta \rightarrow 0$ in the limit that either $\mu^{2} \gg\left|m_{H_{u, d}}^{2}\right|$ or $m_{H_{u}}^{2} \rightarrow m_{H_{d}}^{2}$; in these limits $\tilde{m}$ is identified with $\mu_{\lambda}$. The gray-shaded region is excluded since $\mu^{2}+m_{H_{u}}^{2}<0$ or $\mu^{2}+m_{H_{d}}^{2}<0$ and there is no stable vacuum with a large hierarchy between the weak scale and the supersymmetry breaking scale. In the blue-shaded region, where $\lambda(\tilde{m})_{\text {tree }}>0.01, \tilde{m}$ is predicted to be below a few $10^{9} \mathrm{GeV}$. As we will see in the next section, the Higgsino or sneutrino LSP then gives too large a direct detection rate. However, there is a remarkably large region of parameter space in figure 2 with $\lambda(\tilde{m})_{\text {tree }}<0.003$. 


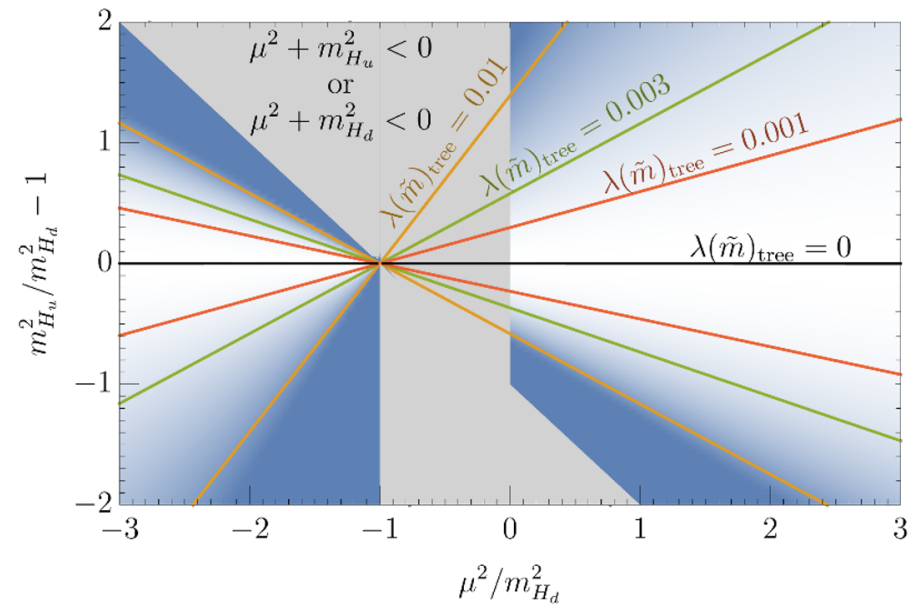

Figure 2. Regions of parameter space showing the smallness of the ISS tree-level prediction for the Higgs quartic coupling at the scale $\tilde{m} . \lambda(\tilde{m})_{\text {tree }}$ is less than $10^{-3}$ if $\mu$ is much greater than $m_{H_{u}}$ and $m_{H_{d}}$, or if $m_{H_{u}}$ and $m_{H_{d}}$ are nearly degenerate. The tree-level prediction is zero when $m_{H_{d}}^{2}=m_{H_{u}}^{2}$, as indicated by the black horizontal line. In the gray region, one of the Higgs doublets has a negative mass squared. With Higgsino or sneutrino LSP, the blue region is excluded by XENON1T.

\section{Direct detection of dark matter}

In this section, we discuss direct detection of the Higgsino or sneutrino LSP dark matter in nuclear recoil experiments and show that detection rates are correlated with SM parameters through the connection between $\tilde{m}$ and $\mu_{\lambda}$. An observable direct detection signal is predicted for top masses below a critical value.

\subsection{Higgsino or sneutrino dark matter}

\subsubsection{Higgsino dark matter}

The neutral components and the charged component of the Higgsino are degenerate in mass in the electroweak symmetric limit. With electroweak symmetry breaking, the charged component becomes heavier than the neutral components by $O(100) \mathrm{MeV}$ via one-loop quantum corrections [25]. The neutral components slightly mix with the bino and the wino and obtain a small mass splitting

$$
\Delta m \sim \frac{g^{2} v^{2}}{M_{2}} \approx 10 \mathrm{keV}\left(\frac{M_{2}}{10^{9} \mathrm{GeV}}\right)^{-1}
$$

The two mass eigenstates are Majorana fermions. For a soft mass scale above $\sim 10^{9} \mathrm{GeV}$, however, the splitting is smaller than the typical nucleon recoil energy of $O(10-100) \mathrm{keV}$, and the Majorana nature does not affect the rate of dark matter signals. Specifically, $Z$ boson exchange leads to the up-scattering of the ligher state into the heavier state, which almost behaves as scattering of a Dirac fermion. 


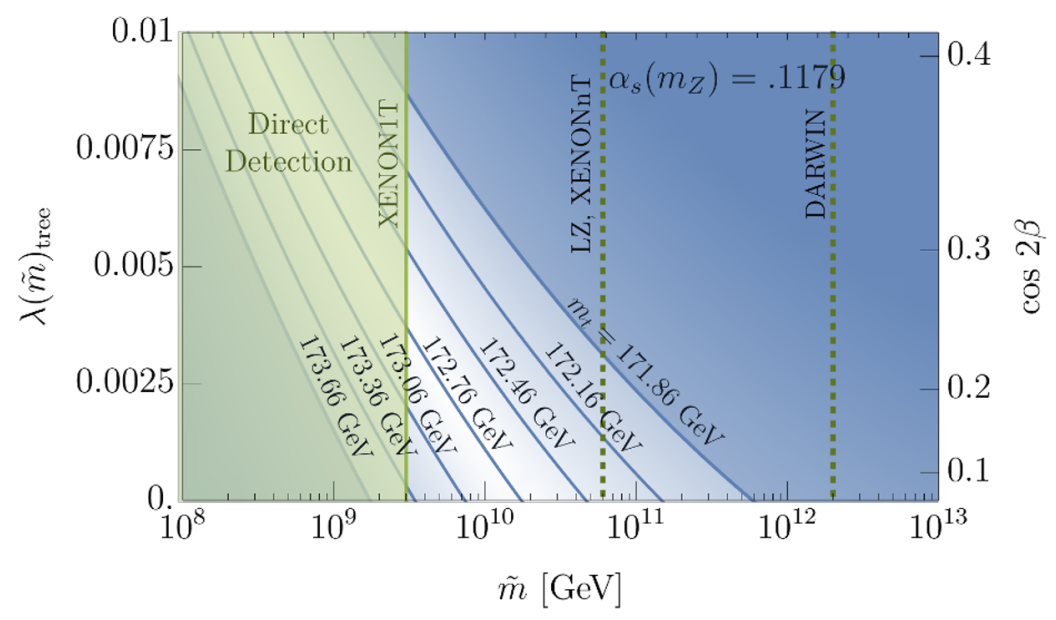

Figure 3. Prediction for the top quark mass as a function of the sparticle mass scale, $\tilde{m}$, and the tree-level Higgs quartic coupling at $\tilde{m}$. Contours of $m_{\text {top }}$ span $3 \sigma$ above and below the current central value for $m_{\text {top }},(172.76 \pm 0.30) \mathrm{GeV}$. For Higgsino or sneutrino LSP dark matter, the green shaded region is excluded by XENON1T and dotted green lines show the sensitivities of future experiments. Values of $m_{t}$ are experimentally disfavored in the dark blue region.

\subsubsection{Sneutrino dark matter}

The sneutrino is lighter than its charged $\mathrm{SU}(2)$ partner because of electroweak symmetry breaking and quantum corrections. The two components of the sneutrino obtain a small mass splitting from the $A$ term of the Majorana neutrino mass term,

$$
\Delta m \sim \frac{A m_{\nu}}{m_{\tilde{\nu}}}
$$

which is negligibly small. Sneutrino dark matter interacts with nucleon via $Z$ boson exchange as a complex scalar field.

If the slepton and squark masses are universal at the unification scale, the sneutrino cannot be the LSP because renormalization running makes the right-handed stau the lightest among them. Non-universality is required for the sneutrino LSP. We note that the sneutrino LSP is consistent with $\mathrm{SU}(5)$ unification, since the sneutrinos and the right-handed sleptons are not unified, and the right-handed down type squarks become heavier than the sneutrinos by renormalization running.

\subsection{Direct detection rate and standard model parameters}

Both Higgsino and sneutrino dark matter scatter with nuclei, with an effective dark matternucleon scattering cross section given by

$$
\sigma_{n}=\frac{G_{F}^{2} m_{n}^{2}}{2 \pi}\left[\frac{(A-Z)-\left(1-4 \sin ^{2} \theta_{W}\right) Z}{A}\right]^{2},
$$

where $G_{F}$ is the Fermi constant, $m_{n}$ is the nucleon mass, $A$ is the mass number, $Z$ is the atomic number, and $\theta_{W}$ is the Weinberg angle. The current constraint by XENON1T [2] 
and the future sensitivities of LZ with an exposure of 15 ton-year, XENONnT with an exposure of 20 ton.year, and DARWIN with an exposure of 1000 ton $\cdot y e a r ~[3-5]$ are given by

$$
\begin{aligned}
\sigma_{n} & <2 \times 10^{-11} \mathrm{GeV}^{-2} \frac{m_{\mathrm{DM}}}{10^{10} \mathrm{GeV}} \\
\sigma_{n} & <1 \times 10^{-12} \mathrm{GeV}^{-2} \frac{m_{\mathrm{DM}}}{10^{10} \mathrm{GeV}} \text { (XZ, XENONnT, future). } \\
\sigma_{n} & <4 \times 10^{-14} \mathrm{GeV}^{-2} \frac{m_{\mathrm{DM}}}{10^{10} \mathrm{GeV}} \text { (DARWIN, future) },
\end{aligned}
$$

which translates into the constraint on and the sensitivity to the Higgsino or sneutrino dark matter mass of

$$
\begin{array}{ll}
m_{\mathrm{DM}}>3 \times 10^{9} \mathrm{GeV} & \text { (XENON1T, current), } \\
m_{\mathrm{DM}}>6 \times 10^{10} \mathrm{GeV} & \text { (LZ, XENONnT future), } \\
m_{\mathrm{DM}}>2 \times 10^{12} \mathrm{GeV} & \text { (DARWIN, future) } .
\end{array}
$$

Once dark matter signals are found in recoil experiments, within the framework of Higgsino or sneutrino dark matter in ISS, the dark matter mass is fixed from the observed signal rates. Since $\lambda(\tilde{m})_{\text {tree }}$ is positive and $m_{\mathrm{DM}}=m_{\mathrm{LSP}}<\tilde{m}$, we obtain a bound on SM parameters including an upper bound on the top quark mass. Conversely, for given SM parameters, $m_{\mathrm{DM}}$ is bounded from above. The prediction for the top quark mass for given $\tilde{m}$ and $\lambda(\tilde{m})_{\text {tree }}$ is shown in figure 3 . The right vertical axis shows $\cos 2 \beta$ corresponding to $\lambda(\tilde{m})_{\text {tree. }}$ For a given $m_{\mathrm{DM}}$, the prediction on $m_{t}$ for $\lambda(\tilde{m})_{\text {tree }}=0$ and $\tilde{m}=0$ can be understood as an upper bound on $m_{t}$. For a given $m_{t}, \tilde{m}$ such that $\lambda(\tilde{m})_{\text {tree }}=0$ in an upper bound on $m_{\mathrm{DM}}$. To obtain those bounds precisely, we include threshold corrections to $\lambda(\tilde{m})$ in the next section.

\section{Including threshold corrections to the Higgs quartic}

The full prediction for $\lambda(\tilde{m})$ in ISS is

$$
\lambda(\tilde{m})=\lambda(\tilde{m})_{\text {tree }}+\delta \lambda(\tilde{m}),
$$

where $\lambda_{\text {tree }}$ is the tree-level result, (2.3), and $\delta \lambda$ the quantum corrections that arise on integrating out heavy sparticles. The largest contributions arise from sparticles with the largest couplings to the light Higgs; hence the most important mass parameters are the masses of the third generation doublet squark $m_{\tilde{q}}$, the third generation up-type squark $m_{\tilde{u}}$, the bino $M_{1}$, the wino $M_{2}$, the heavy Higgs $m_{A}$, and the $A$ term of the top quark yukawa $A_{t}$.

We choose the matching scale to be the lighter of $m_{\tilde{q}}$ and $m_{\tilde{u}}$, which we denote as $m_{-}$. Since quantum corrections are greater than $\lambda_{\text {tree }}$ only for $\tan \beta \simeq 1$, we neglect corrections which vanish in this limit. Using the results in [26], the corrections are given by

$$
\begin{aligned}
32 \pi^{2} \delta \lambda\left(m_{-}\right)= & 3 y_{t}^{4}\left(\ln \frac{m_{\tilde{q}}^{2}}{m_{-}^{2}}+\ln \frac{m_{\tilde{u}}^{2}}{m_{-}^{2}}+2 X_{t} F\left(\frac{m_{\tilde{q}}}{m_{\tilde{u}}}\right)-\frac{X_{t}^{2}}{6} G\left(\frac{m_{\tilde{q}}}{m_{\tilde{u}}}\right)\right) \\
& -\frac{1}{4}\left(g^{\prime 4}+2 g^{\prime 2} g^{2}+\frac{16}{3} g^{4}\right)-\frac{4}{3} g^{\prime 4} f_{1}\left(\frac{M_{1}}{\mu}\right)-4 g^{4} f_{1}\left(\frac{M_{2}}{\mu}\right)-\frac{8}{3} g^{\prime 2} g^{2} f_{2}\left(\frac{M_{1}}{\mu}, \frac{M_{2}}{\mu}\right) \\
& -\left(g^{\prime 4}+2 g^{\prime 2} g^{2}+3 g^{4}\right) \ln \left(\frac{\mu}{m_{-}}\right)+\frac{1}{8}\left(g^{\prime 4}+2 g^{\prime 2} g^{2}+3 g^{4}\right) \ln \frac{m_{A}^{2}}{m_{-}^{2}} .
\end{aligned}
$$



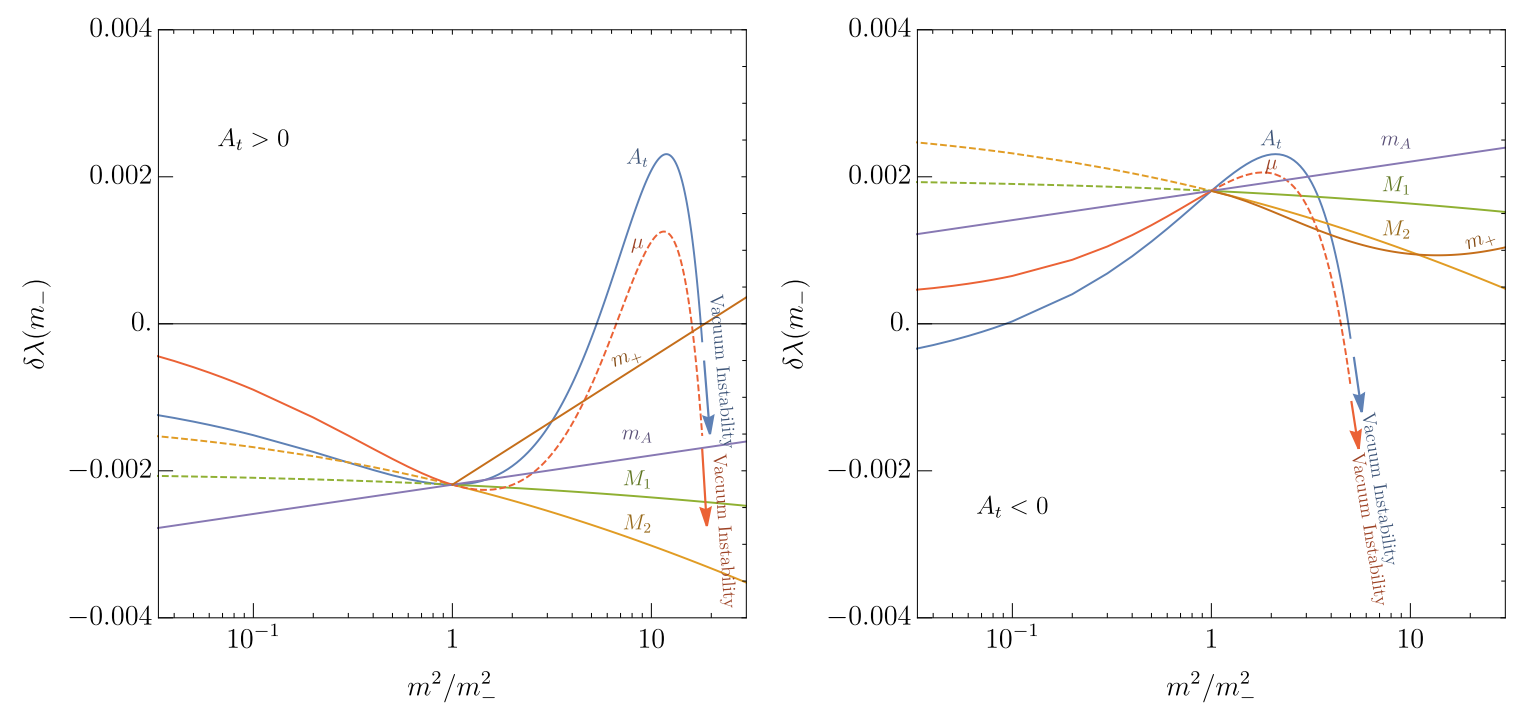

Figure 4. Threshold corrections to the Higgs quartic coupling as a function of sparticle mass parameters. The six curves correspond to $m=\left(A_{t}, \mu, m_{+}, m_{A}, M_{1}, M_{2}\right)$ with the remaining five parameters fixed at $m_{-}=\min \left(m_{\tilde{q}}, m_{\tilde{u}}\right)$. The Higgsino can be the LSP on the solid curves, but is not the LSP on the dashed part of the curves for $\mu, M_{1}$ and $M_{2}$. Left $A_{t}>0$. Vacuum instability occurs when $A_{t}, \mu \gtrsim 4.2 m_{-}$. Right $A_{t}<0$. Vacuum instability occurs when $\left|A_{t}\right|, \mu \gtrsim 2.2 m_{-}$.

Here, $X_{t} \equiv\left(A_{t}-\mu\right)^{2} / m_{\tilde{u}} m_{\tilde{q}}$, and the functions $F, G, f_{1}, f_{2}$ are given by

$$
\begin{aligned}
F(x) & =\frac{2 x \ln x}{x^{2}-1}, \quad G(x)=\frac{12 x^{2}\left(1-x^{2}+\left(1+x^{2}\right) \ln x\right)}{\left(x^{2}-1\right)^{3}}, \\
f_{1}(x) & =\frac{3(x+1)^{2}}{8(x-1)^{2}}+\frac{3(x-3) x^{2} \ln x}{4(x-1)^{3}}, \\
f_{2}(x, y) & =\frac{3(1+x+y-x y)}{8(x-1)(y-1)}+\frac{3 x^{3} \ln x}{4(x-1)^{2}(x-y)}-\frac{3 y^{3} \ln y}{4(y-1)^{2}(x-y)} .
\end{aligned}
$$

They are normalized so that they are unity when the arguments are unity. For a degenerate mass spectrum and negligible $X_{t}, \delta \lambda\left(m_{-}\right) \simeq-0.002$.

In figure 4 , we evaluate eq. (4.2) and show how $\delta \lambda$ varies as a function of sparticle masses. The left and right panels correspond to $A_{t}$ positive and negative, respectively. Each curve corresponds to varying one of $\left(A_{t}, \mu, m_{+}, m_{A}, M_{1}, M_{2}\right)$, while keeping all the others fixed at $m_{-}$. With all these parameters near $m_{-}$, the correction is $\delta \lambda\left(m_{-}\right) \simeq-0.002$ for $A_{t}>0$ or +0.002 for $A_{t}<0$. For $\left|X_{t}\right| \gtrsim 10 m_{-}$, the electroweak vacuum is unstable, as shown by the sudden discontinuation of the $A_{t}$ and $\mu$ curves. The bound on $X_{t}$ from the instability is derived in appendix A. The Higgsino can be the LSP on the solid curves, but is not the LSP on the dashed part of the curves for $\mu, M_{1}$ and $M_{2}$. The slepton mass parameter $m_{\tilde{l}}$ may be taken small enough to give sneutrino LSP anywhere on the lines.

We show contours of the prediction for $m_{t}$ in the $\left(m_{-}, \lambda\left(m_{-}\right)\right)$plane in figure 5 , with the strong coupling constant varied within $\pm 1 \sigma$ uncertainty from its central value in the top and bottom panels. The right axis shows $\cos 2 \beta$ corresponding to $\lambda\left(m_{-}\right)$when $\delta \lambda \ll \lambda_{\text {tree }}$. The lower bound on the dark matter mass from XENON1T is shown in green, and the 

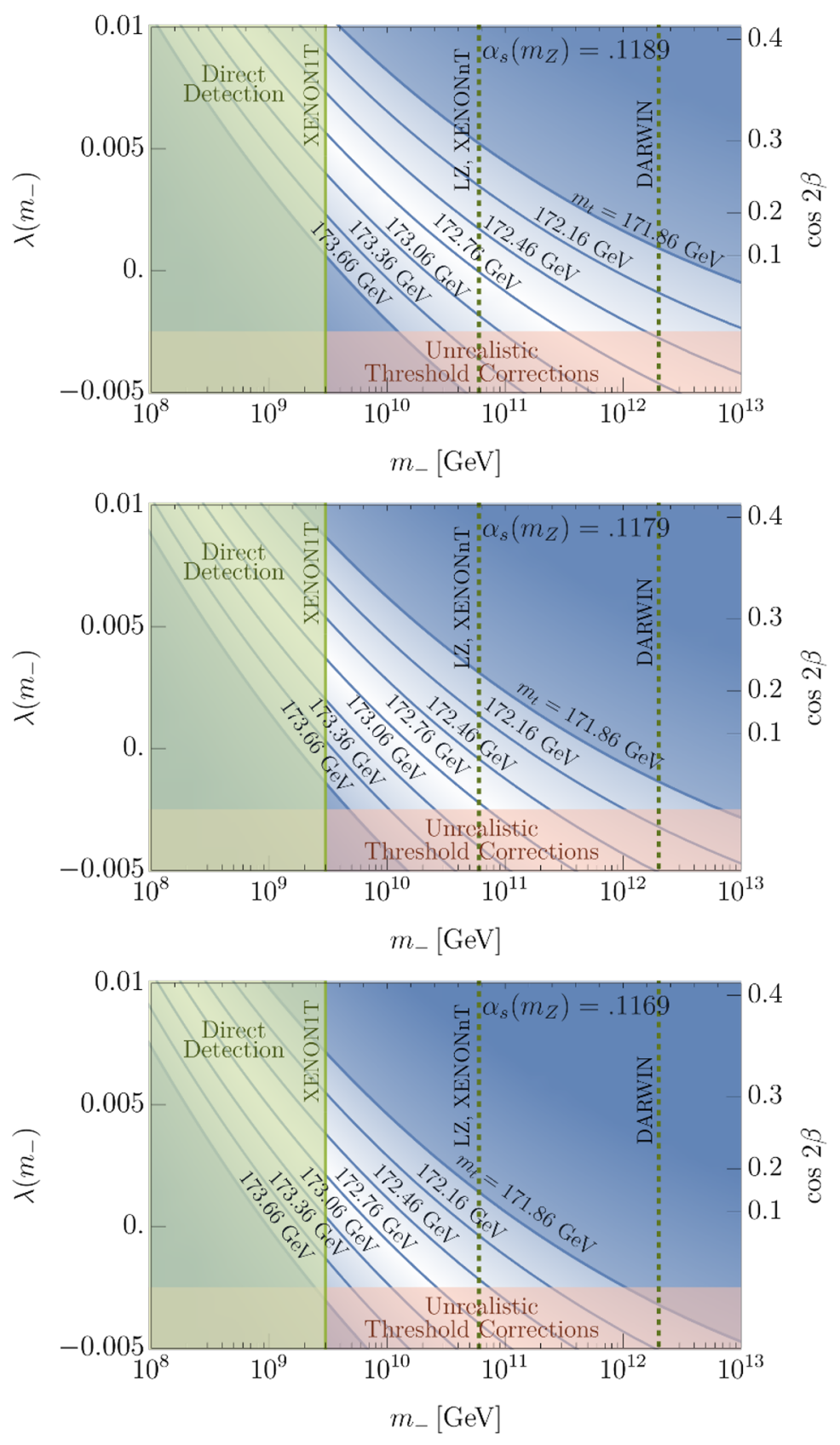

Figure 5. Prediction for the top quark mass as a function of $m_{-}=\min \left(\mathrm{m}_{\tilde{\mathrm{q}}}, \mathrm{m}_{\tilde{\mathrm{u}}}\right)$ and the Higgs quartic coupling at $m_{-}$. Contours of $m_{t}$ span $3 \sigma$ above and below the current central value for $m_{t}$, $(172.76 \pm 0.30) \mathrm{GeV}$. The red shaded region requires unrealistically large negative supersymmetric threshold corrections to the quartic coupling. The green shaded region and the green dotted lines are as in figure 3. Values of $m_{t}$ are experimentally disfavored in the dark blue region. 
lower bound on threshold corrections to $\lambda\left(m_{-}\right)$is shown in red. Together, these bounds require $m_{t} \lesssim 174.2 \mathrm{GeV}$. The reach of the DARWIN experiment, shown by the dashed green line, will strongly limit the top quark mass to $m_{t} \lesssim 172.4 \mathrm{GeV}$, if no signals are found. For the central values of SM parameters, the dark matter mass is required to be below $7 \times 10^{10} \mathrm{GeV}$, and $\mathrm{LZ}$ and XENONnT can cover most of the parameter space.

The bounds on the dark matter and top quark masses may be relaxed by hierarchical sparticle masses. As shown in figure 4, large wino or bino masses give negative threshold corrections to the quartic coupling, thereby relaxing the upper bounds on the top quark mass and the dark matter mass. In figure 6, we show the upper bound on the dark matter mass as a function of the top quark mass or, equivalently, the upper bound on the top quark mass as a function of the dark matter mass. The blue curve is without threshold corrections, the orange curve has threshold corrections for a degenerate mass spectrum with $A_{t} \simeq \mu$, and on the green curve, the degeneracy is lifted by taking $M_{1,2}=\sqrt{10} m_{-}$. With this hierarchy, the upper bound on the dark matter mass is relaxed by a factor of 2 , and that on the top quark mass is relaxed by $100 \mathrm{MeV}$. (Assuming a high mediation scale of supersymmetry breaking, a larger hierarchy is destabilized by quantum corrections from the gauginos to the soft scalar masses.)

In figure 7, the upper bound on the dark matter mass or, equivalently, the upper bound on the top quark mass or the lower bound on the strong coupling constant, is shown. Here we impose $\delta \lambda\left(m_{-}\right)>-0.002$. The current $2 \sigma$ uncertainty of $m_{t}$ and $\alpha_{s}\left(m_{Z}\right)$ are shown by wide bands. The uncertainty of $\alpha_{s}\left(m_{Z}\right)$ can be reduced by a factor of 10 by measurements at future lepton colliders [21] or improved lattice calculations [22]. The uncertainty of $m_{t}$ can be reduced down to few $10 \mathrm{MeV}$ by future lepton colliders [17-20]. At this stage, the theoretical computation of the running of the Higgs quartic coupling should be improved; the most recent computation [6] has a theoretical uncertainty equivalent to the shift of the top quark mass by $100 \mathrm{MeV}$.

\section{Supersymmetry breaking constrained by unification}

In this section, we discuss the quartic coupling at the supersymmetry breaking scale, $\tilde{m}$, starting from boundary conditions at the unification scale $\sim 10^{16} \mathrm{GeV}$. We show that the tree-level quartic coupling is typically $0.001-0.01$.

As we have seen, the quartic coupling at $\tilde{m}$ is small when $m_{H_{u}}^{2} \sim m_{H_{d}}^{2}$. A relation $m_{H_{u}}^{2}=m_{H_{d}}^{2}$ can be naturally realized at a high energy scale by a symmetry relating $H_{u}$ with $H_{d}$, such as a discrete symmetry or $\mathrm{SO}(10)$ gauge symmetry, or a universality of scalar masses. The relation is necessarily destabilized by quantum correction from the top quark Yukawa coupling,

$$
\frac{\mathrm{d}}{\mathrm{d} \ln \mu} m_{H_{u}}^{2}=\frac{3 y_{t}^{2}}{8 \pi^{2}}\left(m_{H_{u}}^{2}+m_{\tilde{q}}^{2}+m_{\tilde{\tilde{u}}}^{2}+A_{t}^{2}\right)+\cdots
$$

where the ellipsis denotes terms independent of the top Yukawa. We compute the renormalization group running of the MSSM from a scale $10^{16} \mathrm{GeV}$ down to $\tilde{m}$ with a UV boundary 


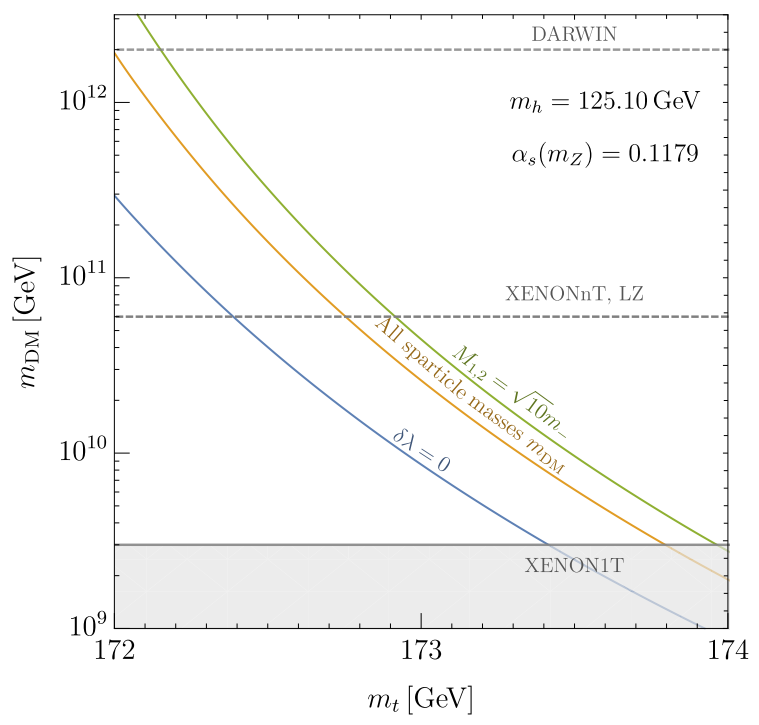

Figure 6. Upper bound on the dark matter mass $m_{\mathrm{DM}}$ as a function of the top quark mass $m_{t}$ for a range of typical threshold corrections. The blue curve shows the bound when the threshold corrections are zero, the orange curve when the sparticle spectra are degenerate $m_{-}$, and in green, when $M_{1,2}=\sqrt{10} m_{-}$. Equivalently, the figure shows an upper bound on $m_{t}$ as a function of $m_{\mathrm{DM}}$.

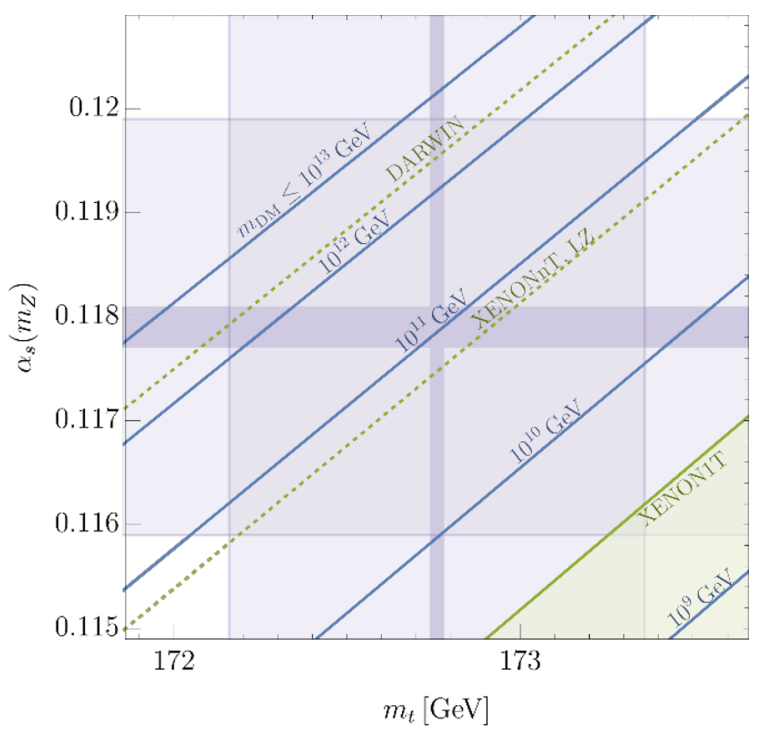

Figure 7. Upper bound on the dark matter mass $m_{\mathrm{DM}}$ as a function of the top quark mass $m_{t}$ and the strong coupling constant $\alpha_{s}\left(m_{Z}\right)$ shown in blue. Equivalently, the figure shows an upper bound on $m_{t}$ as a function of $\alpha_{s}\left(m_{Z}\right)$ and $m_{\mathrm{DM}}$, and a lower bound on $\alpha_{s}\left(m_{Z}\right)$ as a function of $m_{t}$ and $m_{\mathrm{DM}}$. The wider gray bands show the current $2 \sigma$ uncertainties of $m_{t}$ and $\alpha_{s}\left(m_{Z}\right)$, and the narrower bands show the expected future uncertainties. Dark matter direct detection bounds are shown in green. 


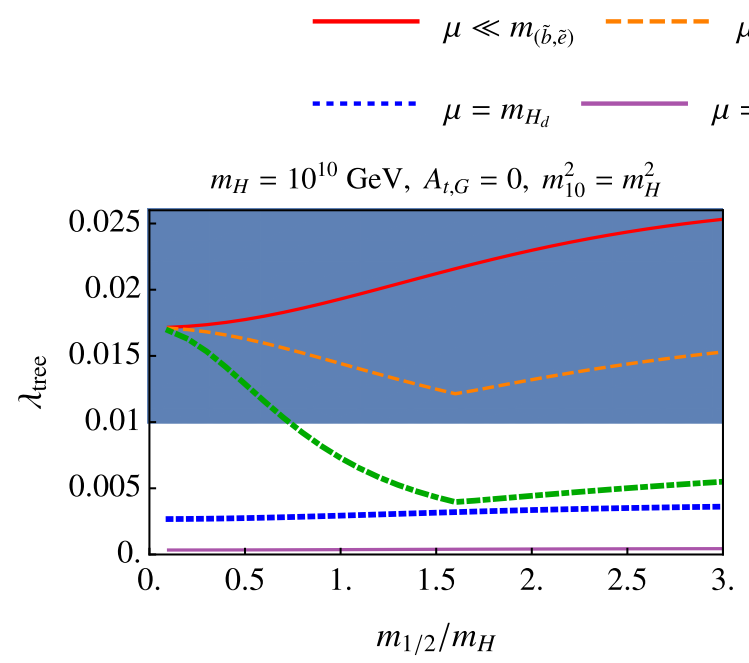

$\mu=m_{(\tilde{b}, \tilde{e})} / 2 \quad----\cdots-\quad \mu=m_{(\tilde{b}, \tilde{e})}$
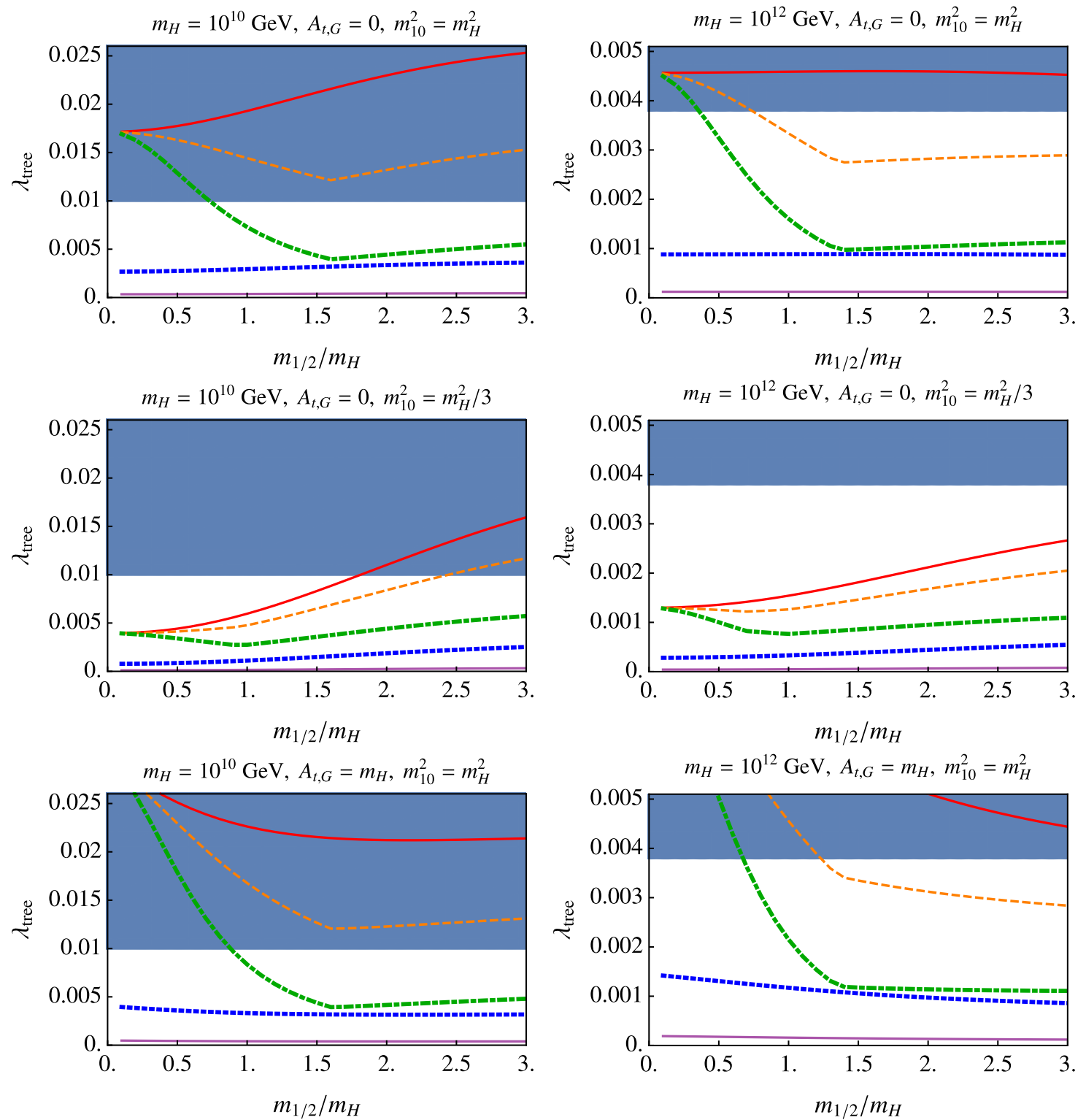

Figure 8. Prediction for the tree-level quartic coupling with a UV boundary condition $m_{H_{u}}=m_{H_{d}}$. In the blue shaded region, reproducing $\lambda\left(m_{-}\right)$requires $m_{t}<171.86 \mathrm{GeV}, 3 \sigma$ away from the central value. Here we impose $\alpha_{s}\left(m_{Z}\right)<0.1189$ and $\delta \lambda\left(m_{-}\right)>-0.002$. 
condition motivated from $\mathrm{SU}(5)$ unification,

$$
\begin{aligned}
m_{H_{u}}^{2} & =m_{H_{d}}^{2}=m_{H}^{2}, & m_{\tilde{q}}^{2} & =m_{\tilde{\tilde{u}}}^{2}=m_{\tilde{\bar{e}}}^{2}=m_{10}^{2}, \quad m_{\tilde{\tilde{d}}}^{2}=m_{\tilde{\ell}}^{2}=m_{5}^{2}, \\
M_{1} & =M_{2}=M_{3}=m_{1 / 2}, & A_{t} & =A_{t, G} .
\end{aligned}
$$

The SM top yukawa coupling is matched to the MSSM top yukawa coupling at $\tilde{m}$ assuming $\tan \beta \simeq 1, y_{t, \mathrm{MSSM}}=\sqrt{2} y_{t, \mathrm{SM}}$. The soft masses $m_{H_{u}}^{2}$ and $m_{H_{d}}^{2}$ at the renormalization scale $\left(10^{10}, 10^{12}\right) \mathrm{GeV}$ are given by the analytic results

$$
\begin{aligned}
& m_{H_{u}}^{2}\left(10^{10} \mathrm{GeV}\right)=0.77 m_{H}^{2}-0.46 m_{10}^{2}-0.12 A_{t, G}^{2}+0.02 m_{1 / 2}^{2}+0.08 m_{1 / 2} A_{t, G}, \\
& m_{H_{d}}^{2}\left(10^{10} \mathrm{GeV}\right)=1.0 m_{H}^{2}+0.19 m_{1 / 2}^{2}, \\
& m_{H_{u}}^{2}\left(10^{12} \mathrm{GeV}\right)=0.86 m_{H}^{2}-0.28 m_{10}^{2}-0.10 A_{t, G}^{2}+0.06 m_{1 / 2}^{2}+0.04 m_{1 / 2} A_{t, G}, \\
& m_{H_{d}}^{2}\left(10^{12} \mathrm{GeV}\right)=1.0 m_{H}^{2}+0.12 m_{1 / 2}^{2} .
\end{aligned}
$$

In figure 8, we show the tree-level quartic coupling as a function of $m_{1 / 2} / m_{H}$ for several representative boundary conditions; the left (right) panels have $m_{H}=10^{10} \mathrm{GeV}$ $\left(10^{12} \mathrm{GeV}\right)$. We fix the renormalization scale to be the matching scale used in the previous section, $m_{-}$, the lighter of $m_{\tilde{q}}$ and $m_{\tilde{u}}$. The boundary condition for $m_{5}^{2}$ is not specified as it does not affect the renormalization group running of $m_{H_{u}}$. Note that the bino, $\tilde{b}$, is the lightest gaugino and the right-handed slepton, $\tilde{e}$, is the lightest scalar in the matter ten-plet. We define $m_{(\tilde{b}, \tilde{e})}$ to be the smaller of $m_{\tilde{b}}$ and $m_{\tilde{e}}$. On the five lines, $\mu$ is fixed to be $\left(\ll m_{(\tilde{b}, \tilde{e})}, m_{(\tilde{b}, \tilde{e})} / 2, m_{(\tilde{b}, \tilde{e})}, m_{H_{d}}, 2 m_{H_{d}}\right)$. As $\mu$ is increased, the tree-level quartic coupling decreases rapidly, as expected from (2.3), (2.4) and figure 2. For large values of $m_{5}^{2}$ the Higgsino is the LSP above the green dot-dashed line, and the region below the line is excluded because at low (high) $m_{1 / 2}$ the LSP is the bino (a charged right-handed slepton). For small values of $m_{5}^{2}$ the tau sneutrino can be the LSP throughout the plane, although at low $\mu$ the Higgsino LSP is also possible. In the blue shaded region, the top quark mass must be below $171.86 \mathrm{GeV}$, more than $3 \sigma$ away from the central value, in order for $\lambda\left(m_{-}\right)$to be consistent with the running of the Higgs quartic coupling. To derive a conservative bound, we take $\alpha_{s}\left(m_{Z}\right)=0.1189,1 \sigma$ above the central value, and $\delta \lambda=-0.002$, the smallest realistic threshold correction.

We see that smaller values of $\lambda_{\text {tree }}$ result for larger $m_{H}$, which gives less running, larger values of $\mu / m_{H}$ and smaller values of $m_{10} / m_{H}$ and $A_{t, G} / m_{H}$. For $m_{H}=10^{12} \mathrm{GeV}$, $\lambda_{\text {tree }}<0.003$ over much of the parameter space. Including threshold corrections, figure 5 shows that this is ideal for consistency with the observed Higgs mass, and requires a low value of the top quark mass. For $m_{H}=10^{10} \mathrm{GeV}, \lambda_{\text {tree }}<0.01$ over much of the parameter space, except at low values of $\mu$, which from figure 5 again shows excellent consistency with the observed Higgs mass, and leads to the expectation that Higgsino/sneutrino dark matter will be discovered at planned experiments.

\section{Cosmological abundance of LSP with intermediate scale mass}

In this section, we discuss how the heavy LSP dark matter can be populated in the early universe. Most of the discussion in this section is applicable to generic heavy dark matter 
with electroweak interactions. Standard freeze-out during the radiation dominated era overproduces the LSP because of its large mass. To avoid this, the reheating temperature of the universe must be smaller than the LSP mass, and the LSP must be produced during the reheating process. We discuss reheating by the inflaton $\phi$, but, if the LSPs produced during inflaton reheating are subdominant, the following discussion also applies to the case where some other particle or condensate dominates the energy density of the universe.

\subsection{Direct decay of the inflaton}

The inflaton can directly decay into sparticles if its mass is more than double the LSP mass. The energy density of the LSP normalized by the entropy density is

$$
\frac{\rho_{\mathrm{LSP}}}{s} \simeq N_{\mathrm{LSP}} \frac{m_{\mathrm{DM}} T_{\mathrm{RH}}}{m_{\phi}}=10^{3} \mathrm{eV} \frac{m_{\mathrm{DM}}}{10^{10} \mathrm{GeV}} \frac{10^{13} \mathrm{GeV}}{m_{\phi}} \frac{T_{\mathrm{RH}}}{\mathrm{MeV}} N_{\mathrm{LSP}},
$$

where $N_{\text {LSP }}$ is the number of LSPs produced per inflaton decay. Because of supersymmetry, $N_{\text {LSP }}$ is at the smallest $O(1)$. When $m_{\phi} \gg m_{\text {DM }}$ and the inflaton dominantly decays into SM charged particles, showering leads to $N_{\mathrm{LSP}} \gg 1$ [27, 28]. Giving the lower bound $T_{\mathrm{RH}}>$ $4 \mathrm{MeV}$ [29-31], it is difficult to produce the correct LSP abundance in this way. Hence, the inflaton mass must be below the sparticle mass scale. (In this case, production of the LSP via scattering among the inflaton decay products and the thermal bath [32-34] is absent.)

\subsection{Production during the inflaton dominated era}

We first derive the evolution of the temperature of the universe. We consider the case where the dissipation of the inflaton occurs by perturbative processes, with dissipation rates given by

$$
\Gamma=\left\{\begin{array}{ll}
\Gamma_{0} & : T<m_{\phi} \\
\Gamma_{0}\left(\frac{T}{m_{\phi}}\right)^{n} & : m_{\phi}<T
\end{array} .\right.
$$

For $T<m_{\phi}$, dissipation is governed by the zero-temperature decay rate $\Gamma_{0}$, while for $m_{\phi}<T$, thermal effects should be taken into account. $n=1$ arises when dissipation is caused by a dimensionless coupling, while $n=-1$ arises when dissipation is caused by a dimension-3 coupling, such as $\phi h h^{\dagger}$.

The dependence of the temperature on the Hubble scale is given by

$$
\begin{aligned}
T_{\mathrm{RH}}<m_{\phi}: & T= \begin{cases}T_{\mathrm{RH}}\left(\frac{H}{H_{\mathrm{RH}}}\right)^{1 / 4} & : T_{\mathrm{RH}}<T<m_{\phi} \\
m_{\phi}\left(\frac{H T_{\mathrm{RH}}^{4}}{H_{\mathrm{RH}} m_{\phi}^{4}}\right)^{1 /(4-n)} & : m_{\phi}<T,\end{cases} \\
m_{\phi}<T_{\mathrm{RH}}: & T=T_{\mathrm{RH}}\left(\frac{H}{H_{\mathrm{RH}}}\right)^{1 /(4-n)},
\end{aligned}
$$

where $H_{\mathrm{RH}}=\sqrt{\pi^{2} g_{*} / 90} T_{\mathrm{RH}}^{2} / M_{\mathrm{Pl}}$ is the Hubble scale at the completion of reheating. We implicitly assumed that the radiation is thermalized, which is not satisfied for small $T_{\mathrm{RH}}$ and/or large $T$. We discuss thermalization while discussing the production of the LSP below. 
Case 1: $\boldsymbol{T}_{\mathrm{FO}}<\boldsymbol{m}_{\phi}<\mathbf{2} \boldsymbol{m}_{\mathrm{DM}}$. During freeze-out, when $T_{\mathrm{FO}}=m_{\phi} / x_{\mathrm{FO}}<m_{\phi}$, radiation is created from the zero-temperature decay of the inflaton and the temperature of the universe is given by the first line of eq. (6.3). Such a case is studied in the literature assuming efficient thermalization [35, 36].

After freeze-out, the LSP number density, normalized by the inflaton energy density, is

$$
\frac{n_{\mathrm{LSP}}}{\rho_{\phi}} \simeq \frac{H_{\mathrm{FO}}}{\langle\sigma v\rangle \rho_{\phi}}=\frac{1}{3\langle\sigma v\rangle H_{\mathrm{FO}} M_{\mathrm{Pl}}^{2}} .
$$

Using $\rho_{\phi} / s \simeq 3 T_{\mathrm{RH}} / 4$ at the completion of reheating, we obtain

$$
\frac{\rho_{\mathrm{LSP}}}{s} \simeq \frac{x_{\mathrm{FO}}^{4}}{4} \sqrt{\frac{90}{\pi^{2} g_{*}}} \frac{1}{4 \pi \alpha_{2}^{2}} \frac{T_{\mathrm{RH}}^{3}}{M_{\mathrm{Pl}} m_{\mathrm{DM}}} \frac{4 \pi \alpha_{2}^{2} / m_{\mathrm{DM}}^{2}}{\langle\sigma v\rangle} .
$$

Here, we assume that radiation thermalizes around the freeze-out temperature. This assumption is valid if $4 \pi \alpha^{2} T_{\mathrm{FO}}>H_{\mathrm{FO}}$, requiring

$$
T_{\mathrm{RH}}>\left[\frac{1}{4 \alpha^{2}} \sqrt{\frac{g_{*}}{90}} \frac{\left(m_{\mathrm{DM}} / x_{\mathrm{FO}}\right)^{3}}{M_{\mathrm{Pl}}}\right]^{1 / 2} \equiv T_{\mathrm{RH}, \mathrm{th}} .
$$

If this condition is violated, the radiation produced from the inflaton does not reach thermal equilibrium by the would-be freeze-out. We expect that the distribution of radiation in this case is close to that after preheating [37, 38]. Since scattering is efficient at lower energies, the lower energy modes are populated. The typical energy of the radiation is below the would-be temperature and the radiation is in an over-occupied state. The energy distribution has a cutoff, above which the scattering is inefficient and the distribution is exponentially suppressed.

For large $m_{\mathrm{DM}}$, the reheating temperature to reproduce the observed abundance from eq. (6.6) is in fact smaller than $T_{\mathrm{RH}, \mathrm{th}}$. Then the LSP abundance is exponentially suppressed and LSPs are under-produced. As $T_{\mathrm{RH}}$ approaches $T_{\mathrm{RH}, \mathrm{th}}$, the LSP production is not suppressed, and the freeze-out picture is applicable. Since $T_{\mathrm{RH}} \sim T_{\mathrm{RH}, \text { th }}$ is larger than that to produce an appropriate amount of LSPs according to eq. (6.6), LSPs are overproduced. Thus, the observed dark matter abundance can be reproduced for $T_{\mathrm{RH}}$ slightly below $T_{\mathrm{RH}, \mathrm{th}}$. We call this scenario non-thermal freeze-in.

The required reheating temperature to produce the observed dark matter abundance by LSP production during reheating is shown in figure 9 . Above the black dashed line, $T_{\mathrm{FO}}<$ $m_{\phi}<2 m_{\text {DM }}$ and the analysis shown above is applicable. To the left of the black dot-dashed line, the LSP abundance is determined by freeze-out, while to the right, the abundance is determined by the exponentially suppressed production just before thermalization.

Case 2: $\boldsymbol{T}_{\mathbf{R H}}<\boldsymbol{m}_{\boldsymbol{\phi}}<\boldsymbol{T}_{\mathbf{F O}}$. For the inflaton mass between $T_{\mathrm{RH}}$ and $T_{\mathrm{FO}}$, the temperature of the universe during freeze-out is given by the second line of eq. (6.3). By a computation similar to that which leads to eq. (6.6), we obtain

$$
\frac{\rho_{\mathrm{LSP}}}{s} \simeq \frac{x_{\mathrm{FO}}^{4-n}}{4} \sqrt{\frac{90}{\pi^{2} g_{*}}} \frac{1}{4 \pi \alpha_{2}^{2}} \frac{T_{\mathrm{RH}}^{3}}{M_{\mathrm{Pl}} m_{\phi}^{n} m_{\mathrm{DM}}^{1-n}} \frac{4 \pi \alpha_{2}^{2} / m_{\mathrm{DM}}^{2}}{\langle\sigma v\rangle} .
$$



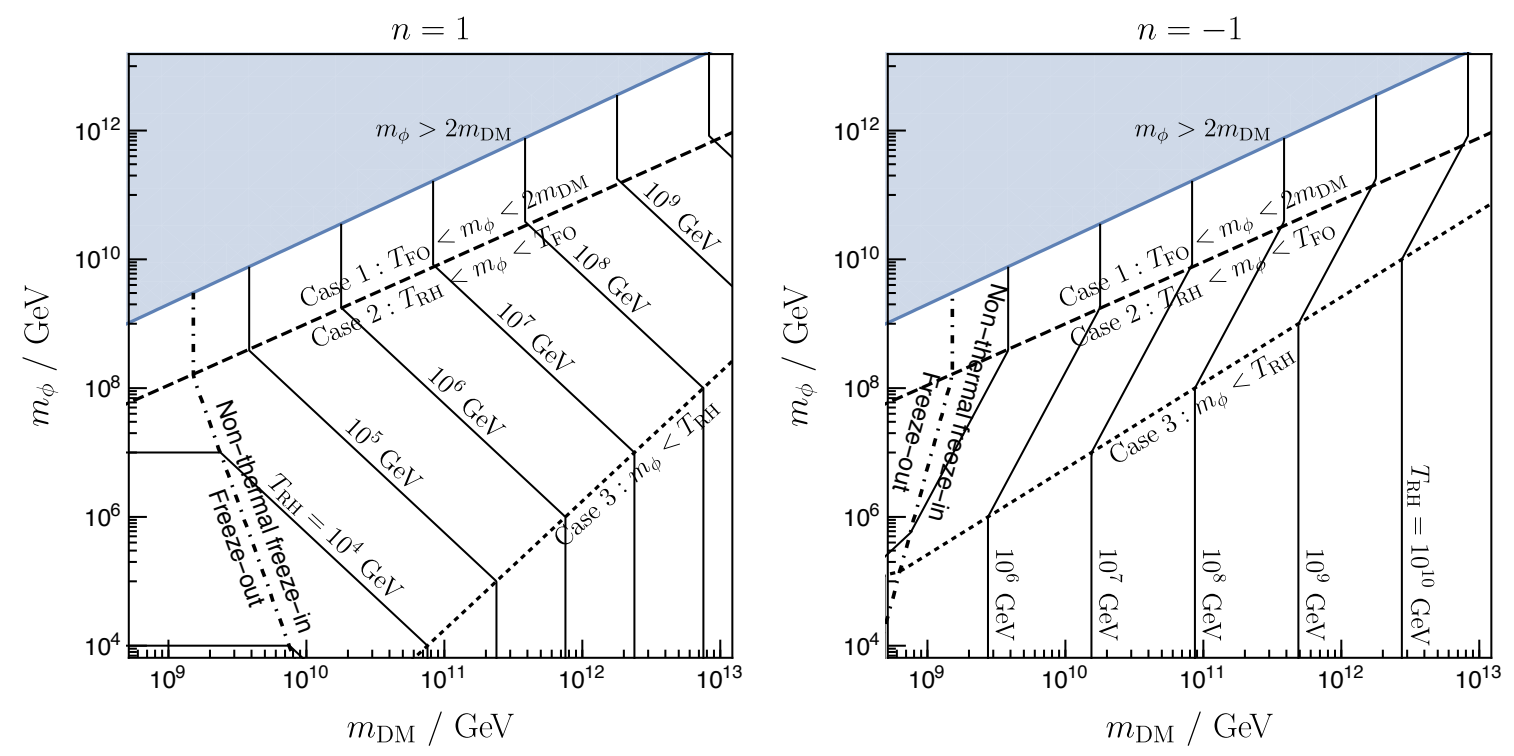

Figure 9. Contours of the reheating temperature $T_{\mathrm{RH}}$ required to produce the observed dark matter abundance by LSP production during reheating. In the blue region, direct decay of the inflaton into sparticles overproduces the LSP. To the right of the dot-dashed line, radiation is not thermalized by the would-be freeze-out, and the LSP production occurs just before the completion of thermalization.

Radiation thermalizes before freeze-out if

$$
T_{\mathrm{RH}}>\left[\frac{1}{4 \alpha^{2}} \sqrt{\frac{g_{*}}{90}} \frac{\left(m_{\mathrm{DM}} / x_{\mathrm{FO}}\right)^{3-n} m_{\phi}^{n}}{M_{\mathrm{Pl}}}\right]^{1 / 2} \equiv T_{\mathrm{RH}, \mathrm{th}} .
$$

The reheating temperature required to produce the observed dark matter abundance is shown in figure 9 . The above analysis is applicable between the dashed and dotted lines.

Case 3: $\boldsymbol{m}_{\phi}<\boldsymbol{T}_{\mathbf{R H}}$. For the inflaton mass below the reheating temperature, the temperature during freeze-out is given by eq. (6.4). The LSP density is given by

$$
\frac{\rho_{\mathrm{LSP}}}{s} \simeq \frac{x_{\mathrm{FO}}^{4-n}}{4} \sqrt{\frac{90}{\pi^{2} g_{*}}} \frac{1}{4 \pi \alpha_{2}^{2}} \frac{T_{\mathrm{RH}}^{3-n}}{M_{\mathrm{Pl}} m_{\mathrm{DM}}^{1-n}} \frac{4 \pi \alpha_{2}^{2} / m_{\mathrm{DM}}^{2}}{\langle\sigma v\rangle} .
$$

Radiation thermalizes before freeze-out if

$$
T_{\mathrm{RH}}>\left[\frac{1}{4 \alpha^{2}} \sqrt{\frac{g_{*}}{90}} \frac{\left(m_{\mathrm{DM}} / x_{\mathrm{FO}}\right)^{3-n}}{M_{\mathrm{Pl}}}\right]^{\frac{1}{2-n}} \equiv T_{\mathrm{RH}, \mathrm{th}} .
$$

The reheating temperature required to produce the observed dark matter abundance is shown in figure 9 . This analysis is applicable below the dotted line.

\subsection{Other possibilities}

It is possible that the maximal temperature of the universe is the reheating temperature. This occurs when reheating is instantaneous, the dissipation rate of the inflaton increases 
towards the end of inflation [39], or a kinematically available decay channel opens suddenly [40]. In this case, the correct LSP abundance is obtained if the reheating temperature is about $m_{\mathrm{DM}} / 10$, so that the LSP production is exponentially suppressed.

The inflaton may also dominantly decay into hidden sector particles with a small branching ratio into the visible sector including the LSP. The entropy of the visible sector can be produced from a moduli field. As long as the mass of the moduli field is smaller than the LSP mass, production of the LSP solely comes from the subdominant decay mode of the inflaton and hence can be suppressed [41].

Finally, the evolution of the early universe may include an era of domination by primordial black holes (PBHs). If the initial Hawking temperature of the PBHs is below $m_{\mathrm{DM}}$, the PBHs emit LSPs only after they lose most of their mass by Hawking radiation into light particles, and the LSP abundance is suppressed. As a result the correct LSP abundance can be obtained for sufficiently large initial PBH masses [42, 43].

\section{Conclusions}

In recent decades, the theoretical and experimental investigations of supersymmetry were focused on weak scale supersymmetry. The discovery of the Higgs with a mass of $125 \mathrm{GeV}$ has revealed a new scale of the SM, the Higgs quartic scale $\mu_{\lambda}=10^{9-12} \mathrm{GeV}$, at which the SM Higgs quartic coupling vanishes. In this paper, we focused on Intermediate Scale Supersymmetry where supersymmetry is broken near the Higgs quartic scale. In this framework, including threshold corrections we found a small SM Higgs quartic coupling for a wide range of supersymmetry breaking parameters. The LSP is a dark matter candidate, and we studied the cases of Higgsino and sneutrino LSP, which scatter with nuclei via tree-level $Z$ boson exchange. Direct detection experiments have already excluded the LSP mass below $3 \times 10^{9} \mathrm{GeV}$, and will probe it up to $10^{12} \mathrm{GeV}$.

The Higgs quartic scale is sensitive to SM parameters. Currently, the uncertainty of the scale is dominated by the top quark mass and the strong coupling constant. We derived an upper bound on the LSP mass as a function of the top quark mass and the strong coupling constant shown in figure 7. Around the central value of SM parameters, dark matter signals should be discovered by near future experiments. Conversely, the figure shows an upper bound on the top quark mass and a lower bound on the strong coupling constant as a function of the LSP mass.

We also discussed how this LSP dark matter may be populated in the early universe. Because of the large LSP mass, the standard freeze-out mechanism overproduces the LSP. We avoid this by taking the reheating temperature after inflation below the LSP mass. We find that the observed dark matter abundance can be obtained during the reheating era, and in most of the parameter space, the inflaton condensate is dissipated by thermal effects during LSP production. We determined the required reheating temperature as a function of the inflaton mass and the LSP mass. Once the LSP mass is fixed by the signal rate at direct detection experiments, the reheating temperature is predicted from the inflaton mass. 


\section{Acknowledgments}

This work was supported in part by the Director, Office of Science, Office of High Energy and Nuclear Physics, of the U.S. Department of Energy under Contracts DE-AC0205CH11231 (LJH), by the National Science Foundation under grant PHY-1915314 (LJH), as well as by Friends of the Institute for Advanced Study (KH).

\section{A Stability bound on a trilinear coupling}

In this appendix, we derive an upper bound on the trilinear coupling between the Higgs and stops from the stability of the electroweak vacuum. We consider the case of $\tan \beta \simeq 1$ and field directions parameterized by

$$
H_{u} \rightarrow \frac{1}{2}\left(\begin{array}{c}
h-H \\
0
\end{array}\right), H_{d} \rightarrow \frac{1}{2}\left(\begin{array}{c}
0 \\
h+H
\end{array}\right), \quad q \rightarrow \frac{1}{\sqrt{2}}\left(\begin{array}{ll}
u & 0 \\
0 & 0 \\
0 & 0
\end{array}\right), \quad \bar{u} \rightarrow \frac{1}{\sqrt{2}}\left(\begin{array}{l}
\bar{u} \\
0 \\
0
\end{array}\right),
$$

where $h, H, u$, and $\bar{u}$ are real fields with potential

$$
\begin{aligned}
V(h, H, q, \bar{u})= & \frac{1}{2} m_{A}^{2} H^{2}+\frac{1}{2} m_{\tilde{q}}^{2} q^{2}+\frac{1}{2} m_{\tilde{u}}^{2} u^{2}-\frac{1}{\sqrt{2}} y_{t} h(A-\mu) u \bar{u}-\frac{1}{\sqrt{2}} y_{t} H(A+\mu) u \bar{u} \\
& +y_{t}^{2}\left(\frac{1}{2} u^{2} \bar{u}^{2}+\frac{1}{4}(h+H)^{2}\left(u^{2}+\bar{u}^{2}\right)\right)+\frac{g^{\prime 2}}{2}\left(\frac{1}{2} h H+\frac{1}{12} u^{2}-\frac{1}{3} \bar{u}^{2}\right)^{2} \\
& +\frac{g^{2}}{2}\left(\frac{1}{2} h H-\frac{1}{4} u^{2}\right)^{2}+\frac{g_{3}^{2}}{24}\left(u^{2}-\bar{u}^{2}\right)^{2} .
\end{aligned}
$$

The renormalization scale of the coupling constants is taken to be around the sparticle mass scale.

The tunneling rate per volume is given by [44]

$$
\frac{\Gamma}{V}=M^{4} \exp ^{-S_{B}},
$$

where $S_{B}$ is a bounce action and $M$ is a pre-factor as large as the typical energy scale associated with the tunneling, which we take to be the sparticle mass scale. To avoid tunneling into another vacuum, we require that $\Gamma / V \times H_{0}^{4}<1$. For sparticle masses around $10^{10} \mathrm{GeV}$, this corresponds to

$$
S_{B}<480 \text {. }
$$

We computed the bounce action using SimpleBounce [45]. For $m_{\tilde{q}}=m_{\tilde{u}}=m_{A} \equiv \tilde{m}$ and $A+\mu=0$, we obtain

$$
|A-\mu|<(3.2-3.3) \tilde{m}
$$

for $\tilde{m}=\left(10^{10}-10^{12}\right) \mathrm{GeV}$. The upper bound excludes large values of $A-\mu$ that would give a negative threshold correction to $\lambda$. For $A+\mu \neq 0$, the bound becomes stronger. Larger $m_{A}$ slightly relaxes the bound, but not enough to enable a negative threshold correction to $\lambda$ from the trilinear coupling. 
Open Access. This article is distributed under the terms of the Creative Commons Attribution License (CC-BY 4.0), which permits any use, distribution and reproduction in any medium, provided the original author(s) and source are credited.

\section{References}

[1] M.W. Goodman and E. Witten, Detectability of certain dark matter candidates, Phys. Rev. D 31 (1985) 3059 [inSPIRE].

[2] XENON collaboration, Dark matter search results from a one ton-year exposure of XENON1T, Phys. Rev. Lett. 121 (2018) 111302 [arXiv:1805.12562] [INSPIRE].

[3] XENON collaboration, Physics reach of the XENON1T dark matter experiment, JCAP 04 (2016) 027 [arXiv : 1512.07501] [INSPIRE].

[4] DARWIN collaboration, DARWIN: towards the ultimate dark matter detector, JCAP 11 (2016) 017 [arXiv : 1606.07001] [inSPIRE].

[5] LUX-ZEPLIN collaboration, Projected WIMP sensitivity of the LUX-ZEPLIN dark matter experiment, Phys. Rev. D 101 (2020) 052002 [arXiv: 1802.06039] [INSPIRE].

[6] D. Buttazzo et al., Investigating the near-criticality of the Higgs boson, JHEP 12 (2013) 089 [arXiv: 1307.3536] [INSPIRE].

[7] L.J. Hall and Y. Nomura, Grand unification and intermediate scale supersymmetry, JHEP 02 (2014) 129 [arXiv: 1312.6695] [INSPIRE].

[8] L.J. Hall, Y. Nomura and S. Shirai, Grand unification, axion, and inflation in intermediate scale supersymmetry, JHEP 06 (2014) 137 [arXiv: 1403.8138] [INSPIRE].

[9] P.J. Fox, G.D. Kribs and A. Martin, Split Dirac supersymmetry: an ultraviolet completion of Higgsino dark matter, Phys. Rev. D 90 (2014) 075006 [arXiv:1405.3692] [InSPIRE].

[10] I. Gogoladze, N. Okada and Q. Shafi, Higgs boson mass from gauge-Higgs unification, Phys. Lett. B 655 (2007) 257 [arXiv:0705.3035] [INSPIRE].

[11] M. Redi and A. Strumia, Axion-Higgs unification, JHEP 11 (2012) 103 [arXiv:1208.6013] [INSPIRE].

[12] L.J. Hall and K. Harigaya, Implications of Higgs discovery for the strong CP problem and unification, JHEP 10 (2018) 130 [arXiv:1803.08119] [INSPIRE].

[13] D. Dunsky, L.J. Hall and K. Harigaya, Higgs parity, strong CP, and dark matter, JHEP 07 (2019) 016 [arXiv: 1902.07726] [INSPIRE].

[14] L.J. Hall and K. Harigaya, Higgs parity grand unification, JHEP 11 (2019) 033 [arXiv: 1905.12722] [INSPIRE].

[15] D. Dunsky, L.J. Hall and K. Harigaya, Dark matter, dark radiation and gravitational waves from mirror Higgs parity, JHEP 02 (2020) 078 [arXiv: 1908.02756] [INSPIRE].

[16] D. Dunsky, L.J. Hall and K. Harigaya, Sterile neutrino dark matter and leptogenesis in left-right Higgs parity, JHEP 01 (2021) 125 [arXiv:2007.12711] [INSPIRE].

[17] K. Seidel, F. Simon, M. Tesar and S. Poss, Top quark mass measurements at and above threshold at CLIC, Eur. Phys. J. C 73 (2013) 2530 [arXiv:1303.3758] [inSPIRE].

[18] T. Horiguchi et al., Study of top quark pair production near threshold at the ILC, arXiv: 1310.0563 [INSPIRE]. 
[19] Y. Kiyo, G. Mishima and Y. Sumino, Strong IR cancellation in heavy quarkonium and precise top mass determination, JHEP 11 (2015) 084 [arXiv:1506.06542] [INSPIRE].

[20] M. Beneke, Y. Kiyo, P. Marquard, A. Penin, J. Piclum and M. Steinhauser, Next-to-next-to-next-to-leading order QCD prediction for the top antitop $S$-wave pair production cross section near threshold in $e^{+} e^{-}$annihilation, Phys. Rev. Lett. 115 (2015) 192001 [arXiv: 1506.06864] [INSPIRE].

[21] TLeP Design Study Working Group collaboration, First look at the physics case of TLEP, JHEP 01 (2014) 164 [arXiv:1308.6176] [INSPIRE].

[22] G.P. Lepage, P.B. Mackenzie and M.E. Peskin, Expected precision of Higgs boson partial widths within the Standard Model, arXiv:1404.0319 [INSPIRE].

[23] M. Cepeda et al., Report from working group 2: Higgs physics at the HL-LHC and HE-LHC, CERN Yellow Rep. Monogr. 7 (2019) 221 [arXiv:1902.00134] [INSPIRE].

[24] B.W. Lee and S. Weinberg, Cosmological lower bound on heavy neutrino masses, Phys. Rev. Lett. 39 (1977) 165 [INSPIRE].

[25] M. Cirelli, N. Fornengo and A. Strumia, Minimal dark matter, Nucl. Phys. B 753 (2006) 178 [hep-ph/0512090] [INSPIRE].

[26] G.F. Giudice and A. Strumia, Probing high-scale and split supersymmetry with Higgs mass measurements, Nucl. Phys. B 858 (2012) 63 [arXiv:1108.6077] [INSPIRE].

[27] Y. Kurata and N. Maekawa, Averaged number of the lightest supersymmetric particles in decay of superheavy particle with long lifetime, Prog. Theor. Phys. 127 (2012) 657 [arXiv:1201.3696] [INSPIRE].

[28] K. Harigaya, T. Lin and H.K. Lou, GUTzilla dark matter, JHEP 09 (2016) 014 [arXiv: 1606.00923] [INSPIRE].

[29] M. Kawasaki, K. Kohri and N. Sugiyama, Cosmological constraints on late time entropy production, Phys. Rev. Lett. 82 (1999) 4168 [astro-ph/9811437] [INSPIRE].

[30] M. Kawasaki, K. Kohri and N. Sugiyama, MeV scale reheating temperature and thermalization of neutrino background, Phys. Rev. D 62 (2000) 023506 [astro-ph/0002127] [INSPIRE].

[31] T. Hasegawa, N. Hiroshima, K. Kohri, R.S.L. Hansen, T. Tram and S. Hannestad, MeV-scale reheating temperature and thermalization of oscillating neutrinos by radiative and hadronic decays of massive particles, JCAP 12 (2019) 012 [arXiv:1908.10189] [INSPIRE].

[32] K. Harigaya, M. Kawasaki, K. Mukaida and M. Yamada, Dark matter production in late time reheating, Phys. Rev. D 89 (2014) 083532 [arXiv: 1402.2846] [INSPIRE].

[33] M.A.G. Garcia and M.A. Amin, Prethermalization production of dark matter, Phys. Rev. D 98 (2018) 103504 [arXiv: 1806.01865] [INSPIRE].

[34] K. Harigaya, K. Mukaida and M. Yamada, Dark matter production during the thermalization era, JHEP 07 (2019) 059 [arXiv: 1901.11027] [INSPIRE].

[35] D.J.H. Chung, E.W. Kolb and A. Riotto, Production of massive particles during reheating, Phys. Rev. D 60 (1999) 063504 [hep-ph/9809453] [INSPIRE].

[36] G.F. Giudice, E.W. Kolb and A. Riotto, Largest temperature of the radiation era and its cosmological implications, Phys. Rev. D 64 (2001) 023508 [hep-ph/0005123] [INSPIRE]. 
[37] R. Micha and I.I. Tkachev, Relativistic turbulence: a long way from preheating to equilibrium, Phys. Rev. Lett. 90 (2003) 121301 [hep-ph/0210202] [INSPIRE].

[38] R. Micha and I.I. Tkachev, Turbulent thermalization, Phys. Rev. D 70 (2004) 043538 [hep-ph/0403101] [INSPIRE].

[39] R.T. Co, E. Gonzalez and K. Harigaya, Increasing temperature toward the completion of reheating, JCAP 11 (2020) 038 [arXiv:2007.04328] [INSPIRE].

[40] T. Fujita and K. Harigaya, Hubble induced mass after inflation in spectator field models, JCAP 12 (2016) 014 [arXiv: 1607.07058] [INSPIRE].

[41] R. Allahverdi, I. Bröckel, M. Cicoli and J.K. Osiński, Superheavy dark matter from string theory, JHEP 02 (2021) 026 [arXiv: 2010.03573] [INSPIRE].

[42] A.M. Green, Supersymmetry and primordial black hole abundance constraints, Phys. Rev. D 60 (1999) 063516 [astro-ph/9903484] [INSPIRE].

[43] T. Fujita, M. Kawasaki, K. Harigaya and R. Matsuda, Baryon asymmetry, dark matter, and density perturbation from primordial black holes, Phys. Rev. D 89 (2014) 103501 [arXiv: 1401.1909] [INSPIRE].

[44] S.R. Coleman, The fate of the false vacuum. 1. Semiclassical theory, Phys. Rev. D 15 (1977) 2929 [Erratum ibid. 16 (1977) 1248] [INSPIRE].

[45] R. Sato, SimpleBounce: a simple package for the false vacuum decay, Comput. Phys. Commun. 258 (2021) 107566 [arXiv: 1908.10868] [INSPIRE]. 